\title{
Microfluidic self-assembly of a combinatorial library of single- and dual-ligand liposomes for in vitro and in vivo tumor targeting
}

Rui $\operatorname{Ran}^{\dagger}$, Haofei Wang ${ }^{\dagger}$, Yun Liu ${ }^{\dagger}$, Yue Hui ${ }^{\dagger}$, Qi Sun ${ }^{\dagger}$, Arjun Seth ${ }^{\dagger}$, David Wibowo ${ }^{\dagger}$, Dong Chen $^{\wedge, \ddagger}$, Chun-Xia Zhao ${ }^{*}, \dagger$

$\dagger$ Australian Institute for Bioengineering and Nanotechnology, The University of Queensland, St. Lucia QLD 4072, Australia

${ }^{\wedge}$ State Key Laboratory of Fluid Power and Mechatronic Systems, Zhejiang University, Zheda Road No. 38, Hangzhou, 310027, China

Institute of Process Equipment, College of Energy Engineering, Zhejiang University, Zheda Road No. 38, Hangzhou, 310027, China

\begin{abstract}
Precise engineering of nanoparticles with systematically varied properties (size, charge surface properties, targeting ligands, etc.) remains a challenge, limiting the effective optimization of nanoparticles for particular applications. Herein we report a single-step microfluidic combinatorial approach for producing a library of single and dual-ligand liposomes with systematically-varied properties including size, zeta potential, targeting ligand, ligand density, and ligand ratio. A targeting ligand folic acid and a cell penetrating peptide TAT were employed to achieve the optimal synergistic targeting effect. In 2D cell monolayer models, the single-ligand folic acid modified liposome didn't show any enhanced cellular uptake, while the incorporation of TAT peptide "switched on" the function of folic acid, and induced significant elevated cellular uptake compared to the single ligand modified liposomes, showing a strong synergistic targeting effect. The folic acid and TAT peptide dual-ligand liposome also demonstrated enhanced tumor penetration as observed using 3D tumor spheroid models. The in vivo study further confirmed the improved tumor targeting and longer tumor retention (up to 72 hours) of the dual-ligand liposomes. Our work not only proved the versatility of this microfluidic combinatorial approach in producing libraries of multifunctional liposomes with
\end{abstract}


controlled properties but also revealed the great potential of the optimized liposome formulation for synergistic targeting effects.

Key words: Dual-ligand liposomes, microfluidics, folic acid, cell penetrating peptides, synergistic effect, tumor targeting

\section{Introduction}

Liposomes are spherical vesicles with an amphiphilic lipid bilayer enclosing an aqueous core[1, 2]. Due to the advantages such as good biocompatibility and good stability, liposomes have gained an intense interest in various fields ranging from vaccine, drug delivery to diagnostics and imaging. From the first FDA-approved nano-drug Doxil[3] to those latest liposome formulations under clinical development $[4,5]$, we have witnessed significant advances in developing lipid-based drug delivery systems for cancer diagnosis and treatment over the past decades. Recently, a lot of effort has been made to develop multifunctional liposomes[6-8]. Through a synergistic manner, these liposomes might address two or more challenges at the same time, such as overcoming multiple barriers to deliver drugs to specific sites, enhancing targeting specificity or increasing circulation time and tumor retention capability.

To achieve desired biological functions, liposomes are usually prepared with some specific ligands. Their biological function can be further enhanced by incorporating two or more ligands at the same time, such as using two targeting ligands or using an active targeting ligand and a cell-penetrating peptide. Ying et. al[9] developed dual-ligand liposomes using two active targeting ligands for transporting the drug across the blood-brain barrier and then targeting brain glioma. Takara et. al[10] used a specific ligand NGR and a cell-penetrating peptide oligoarginine to improve the cellular uptake efficiency through a synergistic effect. These dualligand liposomes[11] are promising candidates to achieve advanced properties and functions such as triggered release, better tumor targeting, and more efficient drug delivery. However, traditional methods for preparing liposomes are mainly based on bulk methods such as thinfilm hydration, freeze-drying, detergent depletion and alcohol injection[12]. These methods always require post-processing steps to homogenize the size, thus often resulting in big batchto-batch variations and poor quality control. To prepare more complex multifunctional liposomes, traditional methods might require further steps to conjugate or incorporate functional groups into the liposomes[13-15], thus leading to increased difficulties in controlling the properties and reproducibility of such liposomes, as well as the associated high manufacture 
cost and time consumed. In addition, as there are so many factors which can affect the in vitro and in vivo properties of multifunctional liposomes, it is essential to prepare a library of liposomes with varied compositions, thus allowing the systematic screening and evaluation. Therefore, it is critical to developing a new platform technology for investigating multifunctional liposomes in a simple, efficient, and reproducible way.

Microfluidic technology is a newly emerging method for the preparation of various nanoparticles due to its better manipulation of the synthesis process[16-18]. To prepare multifunctional liposomes in a single step, a microfluidic hydrodynamic flow focusing (HFF) approach has been developed[19, 20]. This method can provide a well-controlled mixing of the organic solvent which contains the lipids and the aqueous buffer in the microfluidic device, thus precisely controlling the properties of the liposomes such as size, charge, and surface chemistry[21]. The method also allows combinatorial synthesis of libraries of liposomes with systematically-varied properties, which has been considered as an effective way to discover new drugs through high-throughput screening in the pharmaceutical industry. Moreover, this "micro-" preparation process can be easily scaled-up by using high aspect ratio or parallel integrated microfluidic devices[22, 23], which is suitable for massive clinical applications and industrial production.

In this study, we used the hydrodynamic flow focusing method to generate a library of liposomes with varied properties, including different particle size, single ligand, dual-ligand and different ligand densities in a one-step manner. We employed a targeting ligand folic acid (FA) for active tumor targeting and a cell penetrating peptide (TAT) for efficient cell membrane translocation of the liposomes. Their biological functions were evaluated using twodimensional (2D) cell monolayer, three-dimensional (3D) tumor spheroid models, and a tumorbearing mouse model. The work offers a new strategy for preparing libraries of multifunctional liposomes, and the screening of them via various models allows the identification of the best formulation with the optimal biological functions. This strategy could pave the way for the liposome-based pharmaceutical applications.

\section{Materials and methods}

\section{Materials}


TAT peptide with a terminated cysteine (Cys-TAT, CYGRKKRRQRRR, MW 1663) was synthesized by GenScript Corporation (Piscataway, NJ, USA). 1,2-dimyristoyl-sn-glycero-3phosphocholine (DMPC), 1,2-distearoyl-sn-glycero-3-phosphoethanolamine-N[methoxy(polyethylene glycol)-2000] (ammonium salt) (DSPE-PEG 2000 ) were obtained from Avanti Polar Lipids (Alabaster, AL, USA). 1,2-distearoyl-sn-glycero-3-phosphoethanolamineN-[folate(polyethylene glycol)-2000] (DSPE-PEG 2000 -Folate) and 1,2-distearoyl-sn-glycero-3phosphoethanolamine-N-[maleimide(polyethylene glycol)-2000] (DSPE-PEG $2000-\mathrm{Mal}$ ) were obtained from Nanocs (New York, NY, USA). Cholesterol (CHO), histology mounting medium and Accumax solution were purchased from Sigma-Aldrich (St. Louis, MO, USA). Mouse anti-human folate binding protein antibody was purchased from Abcam (Cambridge, UK). Alexa Fluor ${ }^{\circledR} 488$ conjugate goat anti-mouse IgG secondary antibody, Hoechst 33342 and Alexa Fluor ${ }^{\circledR} 647$ Phalloidin were purchased from Invitrogen (Carlsbad, CA, USA). Other chemicals and reagents were of analytical grade.

\section{Synthesis of DSPE-PEG $2000-T A T$}

DSPE-PEG $2000-T A T$ was synthesized by conjugating TAT to the DSPE-PEG $2000-$ Mal through the Michael reaction. Briefly, DSPE-PEG $2000-$ Mal and Cys-TAT (molar ratio $=1: 1.5$ ) were mixed in chloroform/methanol $(\mathrm{v} / \mathrm{v}=2: 1)$, and triethylamine was added as the catalyst. The reaction mixture was protected by nitrogen and then stirred in dark at room temperature for 24 h. The reaction process was monitored by thin-layer chromatography (TLC). After the DSPE$\mathrm{PEG}_{2000}-\mathrm{Mal}$ disappeared based on the TLC, the reaction mixture was evaporated by a rotary evaporation under vacuum. The residue was redissolved in chloroform and filtrated to remove the excess TAT peptide. The purified product was stored at $-20{ }^{\circ} \mathrm{C} .{ }^{1} \mathrm{H}-\mathrm{NMR}$ and MALDI-TOF mass spectrometry were used to verify the successful synthesis of the product.

\section{Preparation of liposomes}

To prepare PEG-Lip (55 mol\% DMPC, 40 mol\% CHO and 5 mol\% DEPE-PEG 2000 ), FA-Lip (55 mol\% DMPC, 40 mol\% CHO, 4 mol\% DEPE-PEG 2000 and 1 mol\% DSPE-PEG $2000-F A$ ), TAT-Lip (55 mol\% DMPC, $40 \mathrm{~mol} \%$ CHO, 4 mol\% DEPE-PEG 2000 and 1 mol\% DSPE$\mathrm{PEG}_{2000}$-TAT) and FA-TAT-Lip (55 mol\% DMPC, 40 mol\% CHO, 3 mol\% DEPE-PEG 2000 , $1 \mathrm{~mol} \%$ DSPE-PEG $2000-\mathrm{FA}$ and $1 \mathrm{~mol} \%$ DSPE-PEG $2000-\mathrm{TAT}$ ), different lipid materials were 
dissolved in $\mathrm{CHCl}_{3}$. After mixing, the chloroform was removed by the rotary evaporator and the lipid film was then re-dissolved in anhydrous isopropanol (IPA). For the fluorescencelabeled liposomes, DiI or DiR was added to the IPA solution together with the lipids at a density of $5 \mu \mathrm{g}$ and $50 \mu \mathrm{g} / \mathrm{mL}$, respectively. The final lipid concentration was adjusted to $2.5 \mathrm{mM}$. For some cell experiments, liposomes with different single- and dual-ligand densities were also prepared, such as $0.5 \mathrm{~mol} \%$ FA modified liposomes (0.5\% FA) or $0.5 \mathrm{~mol} \% \mathrm{FA}$ and $1 \mathrm{~mol} \%$ DSPE-PEG 2000 -TAT modified liposomes (0.5\% FA 1\%TAT) which will be explained in the relevant figure captions.

Liposomes were formed by injecting the lipids into the microfluidic device where this lipids stream was intersected by PBS buffer from side channels. A total flow rate of $28.8 \mu \mathrm{L} / \mathrm{min}$ (corresponding to $0.4 \mathrm{~m} / \mathrm{s}$ ) was used, and flow rate ratios were varied from 3:1 to 16:1 for liposome preparation. The preparation process was conducted at room temperature.

\section{Characterisation of liposomes}

Dynamic light scattering (DLS)

The size, polydispersity index (PDI) and zeta-potential of the liposomes synthesized were determined using a Zetasizer Nano ZS (Malvern Instruments, Worcestershire, UK).

Transmission electron microscopy (TEM)

The morphology of FA-TAT-Lip was observed using a transmission electron microscopy (Jeol, Tokyo, Japan). Samples were dropped onto a copper TEM grid with a carbon film and air-dried at room temperature followed by negative-staining using $1 \%$ uranyl acetate.

In vitro stability in fetal bovine serum (FBS)

Liposomes in 96 well plate were incubated with an equal volume of FBS at $37{ }^{\circ} \mathrm{C}$. At different time points $(0,1,2,4,8,12,24,48 \mathrm{~h})$, the absorbance (A) at $680 \mathrm{~nm}$ was measured using a microplate reader. The relative turbidity at $0 \mathrm{~h}$ was set to be 1 and at other time points was calculated as $\mathrm{A}_{\text {sample }} / \mathrm{A}_{0 \mathrm{~h}}$.

Quantification of FA on liposome surface

The amount of FA was quantified using a fluorescence spectrophotometer. FA-Lip or FA-TATLip were concentrated using centrifugal filters $\left(4000 \mathrm{~g}, 10 \mathrm{~min}, 25^{\circ} \mathrm{C}\right)$ to about $200 \mu \mathrm{L}$ 
followed by the addition of the same volume of $1 \%$ Triton X-100 to de-emulsify the liposomes. Then the absorbance at $380 \mathrm{~nm}$ was recorded by UV-VIS. The amount of FA was calculated using a standard curve. The FA incorporation efficiency (FA IE) was determined as follows:

$$
\text { FA incorporation efficiency }=\frac{\text { The FA amount determined by spectrophotometer }}{\text { The theoretical FA amount }}
$$

Quantification of TAT on liposome surface

TAT peptide on the surface of the liposomes was quantified by RP-HPLC. Briefly, TAT-Lip or FA-TAT-Lip were concentrated by centrifugation ( $4000 \mathrm{~g}, 10 \mathrm{~min}, 25^{\circ} \mathrm{C}$ ) to about $250 \mu \mathrm{L}$ followed by the addition of the same volume of $1 \%$ Triton X-100 to de-emulsify the liposomes. The RP-HPLC was equipped with a Jupiter C18 column $(5 \mu \mathrm{m}, 300 \AA, 150 \mathrm{~mm} \times 4.6 \mathrm{~mm})$ ('Phenomenexe, Torrance, CA, USA) and connected to an LC-10AVP series HPLC system (Shimadzu, Kyoto, Japan). The mobile phase A was $0.1 \%$ (v/v) trifluoroacetic acid (TFA) in water and mobile phase B was $90 \%(\mathrm{v} / \mathrm{v})$ acetonitrile, $0.1 \%$ TFA in water. A linear gradient from 12 to $37 \%$ phase $\mathrm{B}$ in $12 \mathrm{~min}$ at a flow rate of $1 \mathrm{~mL} / \mathrm{min}$ was used. The detector wavelength was set at $214 \mathrm{~nm}$. The TAT incorporation efficiency (TAT IE) was determined as follows:

$$
T A T \text { incorporation efficiency }=\frac{\text { The } T A T \text { amount determined by } R P-H P L C}{\text { The theoretical } T A T \text { amount }}
$$

\section{Cell lines and cell culture}

Murine macrophage cell line (RAW264.7), human ovarian adenocarcinoma cell line (SKOV3) and human breast adenocarcinoma cell line (MCF-7) were purchased from American Type Culture Collection (ATCC). Cell lines were cultured in high glucose DMEM medium supplemented with $10 \% \mathrm{FBS}, 100 \mathrm{U} / \mathrm{mL}$ penicillin and $100 \mathrm{U} / \mathrm{mL}$ streptomycin at $37{ }^{\circ} \mathrm{C}$ in a humidified $5 \% \mathrm{CO}_{2}$ atmosphere.

\section{$2 D$ cell uptake study}

Macrophage cell uptake

RAW 264.7 cells were seeded into a 24 well plate at a density of $2.5 \times 10^{5}$ cells/well and allowed for attachment for overnight. The next day, DiI labeled liposomes with different ligand densities were added into the corresponding wells at a final lipid concentration of $31 \mu \mathrm{M}$. After 
$4 \mathrm{~h}$ incubation at $37{ }^{\circ} \mathrm{C}$, cells were collected, washed three times with PBS, and then resuspended in $0.5 \mathrm{~mL}$ PBS. The fluorescent intensity was measured using Accuri C6 flow cytometer (BD, New Jersey, USA) with the excitation and emission wavelength at 549 and 565 $\mathrm{nm}$, respectively.

Quantitative cell uptake measurement by flow cytometry

SKOV3 or MCF-7 cells were seeded into 24 well plates at a density of $2.5 \times 10^{5}$ cells/well. 24 $\mathrm{h}$ later, DiI labeled liposomes with different ligand density were added to the corresponding wells at a final lipid concentration of $31 \mu \mathrm{M}$. For competitive inhibition group, $0.5 \mathrm{mg} / \mathrm{mL}$ free FA was added to the wells one hour before the addition of liposomes. $4 \mathrm{~h}$ later, cells were collected, washed 3 times with PBS and resuspended in $0.5 \mathrm{~mL}$ PBS for flow cytometry. The excitation and emission wavelengths of 549 and $565 \mathrm{~nm}$, respectively.

Qualitative analysis using confocal laser scanning microscopy (CLSM)

SKOV3 or MCF-7 were seeded into 24 well plates at a density of $1 \times 10^{5}$ cells/well with a 12 $\mathrm{mm}$ diameter of round glass coverslips in each well. $24 \mathrm{~h}$ later, DiI labeled PEG-Lip, FA-Lip, TAT-Lip and FA-TAT-Lip were added to the corresponding wells at a final concentration of $31 \mu \mathrm{M}$ and incubated for $4 \mathrm{~h}$. Then cells were washed with PBS and fixed with $4 \%$ paraformaldehyde for 10 min. After fixing, Alexa Fluor ${ }^{\circledR} 647$ Phalloidin and Hoechst 33342 were used to stain the cytoskeleton and cell nucleus, sequentially. At last, the coverslips were taken out from the wells, mounted to glass slides and observed using CLSM (Zeiss 710, Jena, Germany).

\section{$3 D$ tumor spheroid uptake study}

Formation of 3D tumor spheroids

$5 \times 10^{3}$ cells/well SKOV3 or MCF-7 were seeded into 96 well plate in which each well was precoated with $50 \mu \mathrm{L} 2 \%$ low melting point agarose to prevent adherence of the cells. Plates were incubated at $37{ }^{\circ} \mathrm{C}$ in a humidified $5 \% \mathrm{CO}_{2}$ atmosphere. After about 7 days, spheroids with uniform size were selected for experiments.

Tumor spheroid uptake using CLSM

Tumor spheroids were incubated with DiI labeled PEG-Lip, FA-Lip, TAT-Lip and FA-TATLip (synthesized with a flow rate ratio of $8: 1$ ) for $4 \mathrm{~h}$. Then the spheroids were collected, 
washed 3 times with PBS and immersed in $4 \%$ paraformaldehyde for CLSM analysis. A layerby-layer scanning was used to detect the fluorescent signal of the tumor spheroids in each layer and a Zen (blue edition) software was used to analyze and create the 3D images.

Tumor spheroid uptake using flow cytometry

Tumor spheroids were incubated with DiI labeled PEG-Lip, FA-Lip, TAT-Lip and FA-TATLip (synthesized with an FRR of 8:1) for $4 \mathrm{~h}$. Then 10 spheroids for each group were gathered and treated with $200 \mu \mathrm{L}$ Accumax solution for $30 \mathrm{~min}$ at $37^{\circ} \mathrm{C}$. After dissociation, cells were washed three times with PBS and then analyzed using a flow cytometry with the excitation and emission wavelength at 549 and $565 \mathrm{~nm}$, respectively.

\section{Biodistribution and tumor targeting study}

To establish a tumor model, nude Balb/C mice weighing around 18 grams were injected with $1 \times 10^{7}$ of SKOV3 cells subcutaneously at the left armpit. Biodistribution study begins when tumor size reached about $0.5 \mathrm{~cm}$ in diameter.

The tumor-bearing mice were randomly divided into 4 groups with 8 mice for each group. DiR loaded liposomes were intravenously injected through the tail vein at a dose of $100 \mu \mathrm{g} \mathrm{DiR} / \mathrm{kg}$. At the time points $2,6,12,24,48$ and $72 \mathrm{~h}$ after injection, mice were imaged using an in vivo MS FX Pro instrument (Bruker Corporation, Billerica, MA, USA), fluorescent signal and Xray signal were obtained. On the other hand, one mouse from each group at time points 6,12 , 24, 48 and $72 \mathrm{~h}$ was euthanized, and organs (hearts, livers, spleens, lungs and kidneys) and tumors were collected for ex vivo imaging. The mean fluorescent signal intensity at the tumor site in the whole animal biodistribution results was semi-quantified using Image-J. The ex vivo tumor-to-liver fluorescent signal intensity ratio was performed as the mean fluorescent signal intensity of the tumor (whole tumor fluorescent signal intensity semi-quantified by Image-J divided by the weight of the tumors) divided by the mean fluorescent signal intensity of the liver (whole liver fluorescent signal intensity semi-quantified by Image-J divided by the weight of the livers).

Statistical analysis 
Statistical analysis was performed by the one-way ANOVA for multiple groups, and $p$ value $<0.05,<0.01$ and $<0.001$ were marked as $* * *$ and $* * *$, respectively.

\section{Results and discussion}

We used a one-step process to prepare liposome libraries of PEGylated liposomes, singleligand liposomes (FA modified liposomes and TAT modified liposomes), and dual-ligand liposomes (FA and TAT co-modified liposomes), with different size, the same charge, and different ligand density. We used 1,2-dimyristoyl-sn-glycero-3-phosphocholine (DMPC) and cholesterol as the basic liposome components and DSPE-PEG 2000 as the PEGylated lipid, DSPE-PEG 2000 -FA and DSPE-PEG 2000 -TAT were incorporated to make surface-modified liposomes. In modern nanocarrier design, PEGylation is indispensable as it prolongs in vivo circulation time by reducing the reticuloendothelial system (RES) clearance[24]. FA is able to recognize those cells with elevated expression of the folate receptor, which is expressed on the surface of many cancer cells[25]. TAT is a short cationic peptide derived from the transactivator protein of human immunodeficiency virus and has been demonstrated capable of efficiently translocating cargoes across cell membranes such as nucleic acids, siRNA, protein, chemotherapy agents, and nanosized drug delivery vesicles[26].

\section{Synthesis of DSPE-PEG 2000 -TAT}

TAT peptide is covalently conjugated to the distal end of DSPE-PEG 2000 through the Michael addition reaction between cysteine and maleimide[27, 28]. The reaction process was monitored by a thin-layer chromatography. $4.5 \mathrm{mg}$ of DSPE-PEG $2000-\mathrm{Mal}$ was reacted with $5.3 \mathrm{mg}$ of Cys-TAT and produced $6.4 \mathrm{mg}$ of DSPE-PEG $2000-\mathrm{TAT}$ with a yield of $75 \%$. In the ${ }^{1} \mathrm{H}$ NMR spectra (Fig. S1), the peak 1 (1.013-0.756 ppm) and peak 2 (1.317-1.215 ppm) correspond to $\mathrm{CH}_{3}$ and $-\mathrm{CH}_{2}-$ of DSPE, respectively, and the peak 5 (3.793-3.525 ppm) for $-\mathrm{CH}_{2}-$ of $\mathrm{PEG}_{2000}$. The peak 3 and peak 4 which belong to TAT are still observed in DSPE-PEG $2000-T A T$, confirming the successful conjugation of TAT to DSPE-PEG 2000 . The mass spectrometer shows that the observed molecular weight (4578 Da) is in accordance with the theoretical molecular weight (4605 Da), confirming the successful synthesis of DSPE-PEG $2000-T A T$ (Fig. S1). 
Liposome preparation in microfluidic devices

Fig. 1A shows the microfluidic synthesis of liposomes. The microfluidic device has a nanoprecipitation channel with a width of $20 \mu \mathrm{m}$, a height of $60 \mu \mathrm{m}$ and a length of $1 \mathrm{~cm}$. The lipid-containing isopropanol is injected into the central channel of the microfluidic device and is squeezed by PBS buffer at the junction from two sides. During the nanoprecipitation process, the solubility of the lipids decreases and the lipids will gradually precipitate and self-assemble into liposomes. The morphology of the liposomes synthesized was imaged using TEM; liposomes are observed as hollow and spherical shape vesicles (Fig. 1B). To systematically investigate the biological functions of the liposomes, we not only synthesized dual-ligand liposomes (FA-TAT-Lip) but also PEGylated liposomes (PEG-Lip), single-ligand FA modified liposomes (FA-Lip) and single-ligand TAT modified liposomes (TAT-Lip) as comparisons (Fig. 1C).

A

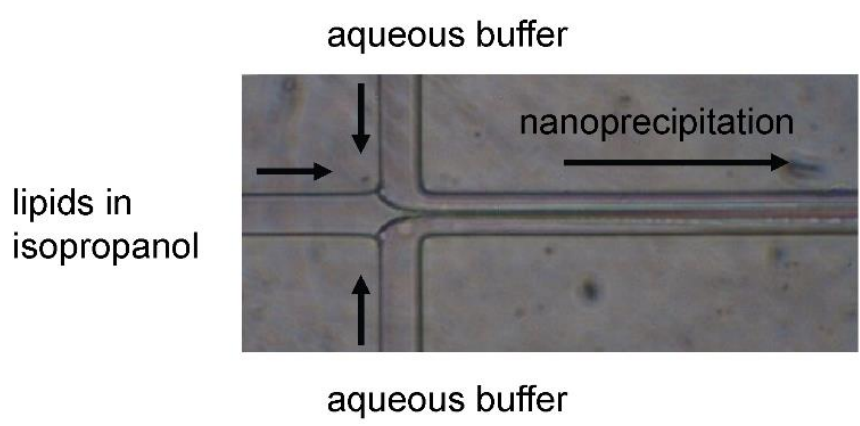

B

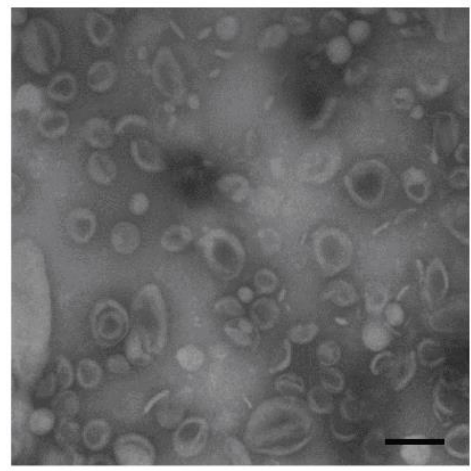

C

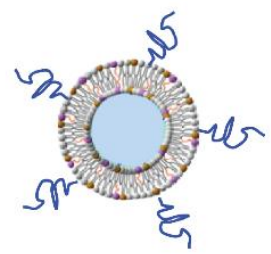

PEG-Lip

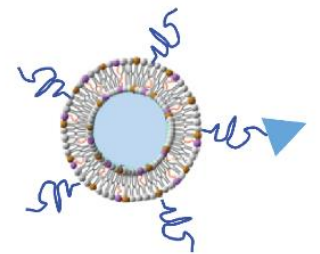

FA-Lip

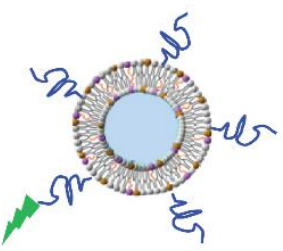

TAT-Lip

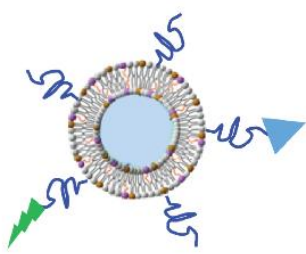

FA-TAT-Lip

Fig. 1. (A) Snapshot of the synthesis of liposomes by nanoprecipitation. Liposomes are selfassembled when the lipid stream is intersected by the aqueous buffer from the side channels. (B) TEM image of the FA-TAT-Lip synthesized by microfluidic method, observed as round hollow spherical vesicles. Scale bar represents $100 \mathrm{~nm}$. (C) Liposomes varied with tunable functional groups by grafting designed chemical groups on lipids, representing PEG-Lip, FA-Lip, TAT-Lip and FA-TAT-Lip. PEG-Lip ( 55 mol\% DMPC, 40 mol\% CHO and 5 mol\% DEPE-PEG 2000 ), FA-Lip ( 55 mol\% DMPC, 40 mol\% CHO, 4 mol\% DEPE-PEG 2000 and 1 mol\% DSPE-PEG $2000-$ FA), TAT-Lip (55 mol\% DMPC, 40 mol\% CHO, 4 mol\% DEPE-PEG 2000 and 1 mol\% DSPE-PEG 2000 -TAT) and FA-TAT-Lip (55 mol\% DMPC, 40 mol\% CHO, 3 mol\% DEPE-PEG 2000,1 mol\% DSPE-PEG $2000-F A$ and 1 mol\% DSPE-PEG $\left.{ }_{2000}-T A T\right)$. 
Tuneable liposome size and surface properties

We control the size of the liposomes in order to achieve the best blood circulation time and tumor accumulation. The liposome's diameter of around or below $100 \mathrm{~nm}$ has been proved to be the optimal size for more effective blood-to-tumor transfer and longer retention in tumor tissue[29]. Microfluidic technology is able to tune the size of liposomes by simply adjusting one of the key parameters - the flow rate ratio (FRR, defined as the volumetric flow rate of the PBS to the volumetric flow rate of the lipid stream). We tune the FRR from 3:1 to 16:1 while the flow velocity of the fluid stream in the liposome nanoprecipitation channel is kept at 0.4 $\mathrm{m} / \mathrm{s}$, corresponding to a total volumetric flow rate of $28.8 \mu \mathrm{L} / \mathrm{min}$. As shown in Fig. $2 \mathrm{~A}$, the sizes of the FA-TAT-Lip smoothly decrease from $\sim 180$ to $\sim 60 \mathrm{~nm}$ as the FRR increases from 3:1 to $16: 1$. At the same time, the polydispersity index (PDI) could keep relatively low during this process (Fig. S4), indicating the uniformity of the liposomes. PEG-Lip, FA-Lip, and TATLip also show the same trend as the FRR increases (Fig. S2, Fig. S3). A non-equilibrium kinetic model could explain the formation of the liposomes through a two-step process in the microfluidic device, i.e. the self-assembly of lipid into planar lipid bilayers, and the bending and closing of the disc to form spherical vesicles. Therefore, the liposome size is dominated by two parameters, i.e. the lipid disc growth time and the lipid disc closure time. When the FRR is high, the alcohol stream quickly mixes with PBS, leading to both a shorter growth time[30] and decreased closure time[31], and thus a smaller liposome size. 
A

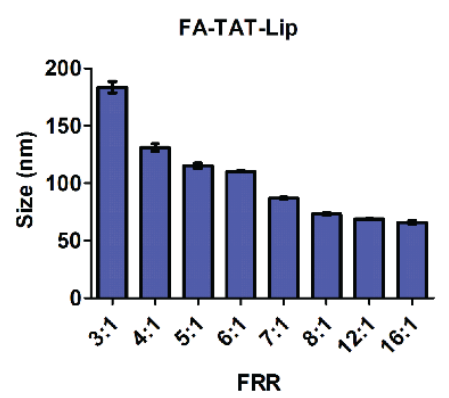

D

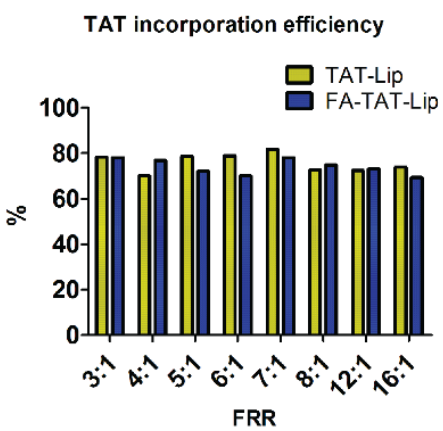

B

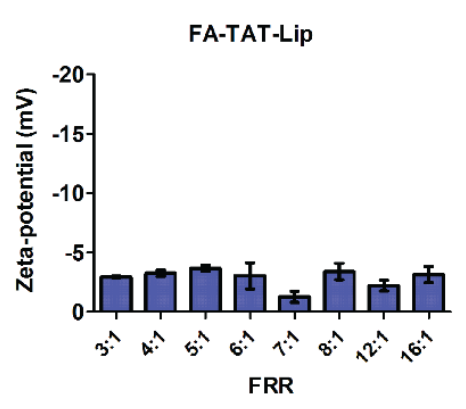

$E$

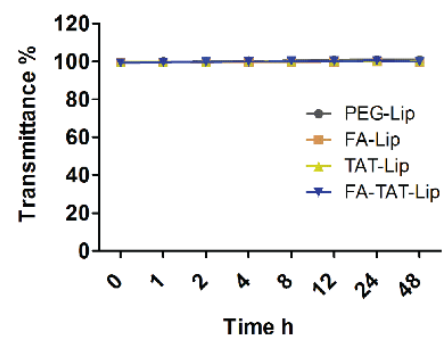

C

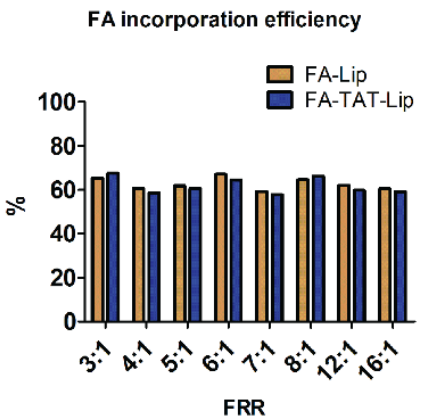

$\mathrm{F}$

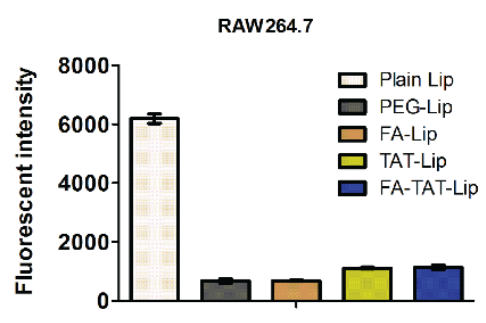

Fig. 2. (A) The correlation between the size and the FRR (flow rate ratio, defined as the volumetric flow rate of the aqueous buffer to that of the organic phase). The size of the liposomes decreases as the high FRRs decrease the lipid disc growth time and the lipid disc closure time. (B) The zetapotentials of FA-TAT-Lip under different FRRs. The incorporation efficiency of the ligand of FA (C) and the ligand of TAT (D), defined as the ligand recovery rate after the nanoprecipitation process. (E) The variation of transmittance of liposomes in the presence of FBS up to $48 \mathrm{~h}$, indication a good serum stability. (F) Flow cytometry result of the RAW 264.7 uptake of Dil labeled liposomes, showing a low macrophage capture compared to non-pegylated liposomes.

Zeta-potential plays a critical role in regulating nanoparticles' circulation time and cellular uptake. Strong negative or positive zeta-potential might lead to elevated macrophage uptake and thus shortened blood circulation time[32]. The zeta-potential variation of FA-TAT-Lip is shown in Fig. 2B. As can be seen, although the liposome sizes are different (different FRR), the zeta-potential could keep constant at slightly negative. Other liposomes such as PEG-Lip, FA-Lip, and TAT-Lip also show a slightly negative zeta-potential despite their different lipid compositions (Fig. S3). This result suggests that the charge remains stable independent of the flow rate ratios (FRRs). So the FRR exhibits a negligible effect on the surface properties of the liposomes. The surface properties can be controlled by using different initial lipid compositions such as anionic or cationic lipids to prepare liposomes with negative or positive zeta-potential, respectively, which has been achieved in our previous study [33]. Notably, TAT-Lip shows 
less negative zeta-potential than other liposomes, demonstrating the incorporation of the cationic TAT peptide onto the liposome surface. The introduction of PEG to the liposomes might shield some original charges of the lipids or the functional materials, leading to a more neutralized or slightly negative surface close to PEG. Therefore, although the incorporation of a cationic peptide TAT, the zeta-potential of TAT-Lip and FA-TAT-Lip remains approximately zero or slightly negative.

Another critical surface property is the ligand density on the surface of the liposomes as it will directly affect the cellular uptake efficiency. We quantified the ligand density on the surface of the liposomes after microfluidic synthesis. The incorporation efficiencies of FA and TAT were measured by UV-VIS and HPLC, respectively[34, 35] (Fig. 2C and Fig. 2D). FA has an average efficiency of $\sim 62.7 \%$ for FA-Lip, and $\sim 61.8 \%$ for FA-TAT-Lip, respectively (which means $\sim 62.7 \%$ and $\sim 61.8 \%$ of the initial DSPE-PEG $2000-F A$ added are successfully incorporated to the surface of FA-Lip and FA-TAT-Lip, respectively). Compared to FA, the TAT peptide has a higher incorporation efficiency ( $75.9 \%$ for TAT-Lip and $\sim 74.1 \%$ for FATAT-Lip). The incorporation efficiency of either FA or TAT could keep constant despite how the liposome size (FRR) varies. We also find that although the initial lipids composition for synthesizing FA-Lip and FA-TAT-Lip are different, the final FA incorporation efficiencies remain very similar, which might imply that the incorporation efficiency is only depended on the innate property of the ligand itself. This is also true for TAT peptide. It should be noted that liposomes have an inner and an outer- lipid layer, so the ligands are not $100 \%$ on the outerlayer of the liposome surface $[36,37]$. Considering the formation process of liposomes in microfluidics through the formation of lipid bilayer discs, on which the ligands, as well as other lipid materials, are evenly distributed, when these discs grow and eventually form liposomes, the ligand density on the inner- or outer- layer of the liposome surface should be the same. So theoretically, $50 \%$ of all the ligands would be on the outer-layer of the liposome surface (which could mediate cell binding and uptake), while the other $50 \%$ would be on the inner-layer of the liposome surface towards the water core. Therefore, the accessible ligands on the liposome outer surface should be half of the total ligand density we quantified. In our following in vitro and in vivo experiments, the ligand density represents the total density. The ligands incorporation efficiency test may help us to better understand the interactions between different lipid components when self-assembling into liposomes, and serve as a guide for novel ligand modified liposome design. 
The liposomes synthesized using the FRR of 8:1 have a diameter of $\sim 70 \mathrm{~nm}(67.9 \pm 2.0,72.8$ $\pm 1.8,66.0 \pm 0.7$ and $73.2 \pm 1.6 \mathrm{~nm}$ for PEG-Lip, FA-Lip, TAT-Lip, and FA-TAT-Lip, respectively), and therefore are selected for the following in vitro and in vivo studies. These liposomes also possess a good serum stability as they do not form aggregates up to $48 \mathrm{~h}$ (Fig. 2E). The macrophage uptake experiments of (Fig. 2F) show that these PEGylated liposomes have a lower macrophage capture compared to non-PEGylated liposomes (Plain-Lip)[33], indicating a good immune evasion, which is a prerequisite for in vivo application.

2D tumor cell monolayer uptake

We used folate receptor (FR) overexpressed SKOV3 cells (as proved by antibody binding experiments, shown in Fig. S5A) for the screening of the liposome formulations. As a comparison, FR negative MCF-7 cells were also employed. The enhanced uptake efficiency of dual-ligand liposomes with 1\% FA and 1\% TAT co-modified surface (FA-TAT-Lip) is shown in Fig. 3A. We observe that FA-TAT-Lip had a $66 \%$ increased uptake than $1 \%$ TAT modified TAT-Lip, and 3.2 times increased uptake than 1\% FA modified FA-Lip. A detailed systematic screening of the uptake efficiency of liposomes is shown in Fig. S6. $1 \mathrm{~mol} \%$ FA and $1 \mathrm{~mol} \%$ TAT modified liposomes have an obvious higher cellular uptake efficiency while a further increase of the FA no longer promotes the cellular uptake efficiency. Therefore liposome formulated with $1 \mathrm{~mol} \% \mathrm{FA}$ and $1 \mathrm{~mol} \%$ TAT is identified (FA-TAT-Lip) as the optimized formulation and is used for the following experiment. The involvement of free FA decreases the uptake efficiency of FA-TAT-Lip to the level of TAT-Lip, indicating the FR mediated cell internalization pathway. This result illustrates that the PEGylation of liposomes, which is essential for long circulation time and in vivo applications, decreases the uptake significantly because of the steric effect of the PEG hindering the interaction between the liposomes with the tumor cells. PEGylation even hinders the interactions between FA-Lip and the cells as FALip could not show an increased cellular uptake when using 1 mol\% FA. In fact, our previous study demonstrated that further increasing the FA density to 4 mol\% could obviously enhance the cellular uptake[33]. Notably, although $1 \mathrm{~mol} \% \mathrm{FA}$ alone seems insufficient in elevating the cellular uptake, the addition of TAT not only promotes the unspecific cell entry but also "switches on" or reinforces the ability of FA, showing a strong synergistic effect. The uptake efficiency was also qualitatively observed using confocal laser scanning microscopy (CLSM) (Fig. 3B). Consistent with the flow cytometry results, both PEG-Lip and FA-Lip do not show 
much cellular uptake by SKOV3 cells, as indicated by the low fluorescence intensity. In contrast, the TAT-Lip displays a much stronger fluorescent intensity, which is further enhanced when using FA-TAT-Lip, demonstrating the synergistic effect. The DiI signal of the FA-TATLip is mainly distributed in the cytoplasm and the perinuclear region, indicating that the liposomes are preferably accumulated in the cytoplasm. However, some fluorescent signal could also be observed in the cell nuclei, showing that TAT peptide can facilitate the entry of liposomes into the nucleoplasm, which is in consistent with some previous studies[38]. As a comparison, the uptake of the liposomes on the FR negative MCF-7 cells is shown in Fig. 3C and Fig. 3D. We could observe that the uptake efficiency of FA-TAT-Lip is similar to that of the TAT-Lip, further proving that the synergistic effect is FR mediated. The results prove that the TAT peptide is very effective in improving cell internalization, but it is unspecific. Therefore, the combination of FA and TAT takes advantage of the unique properties of these two ligands, i.e. enhancing the liposomes' targeting capability and increasing the cellular uptake efficiency, and at the same time reducing the ligand density required, through a synergistic effect. 
A

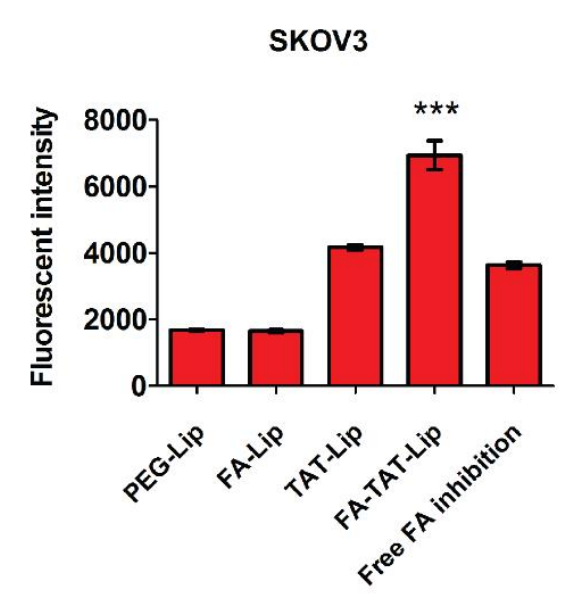

C

MCF-7

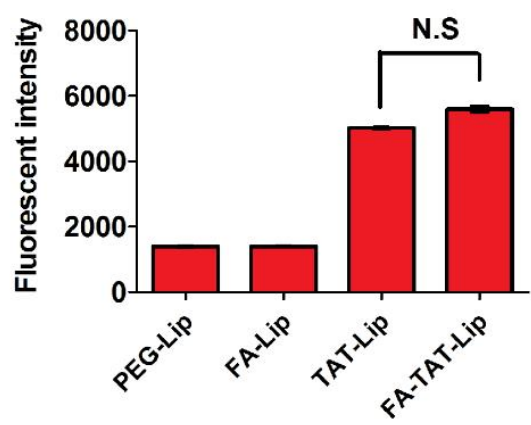

$\mathrm{B}$
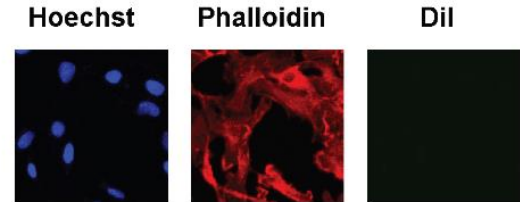

Overlay

FA-Lip
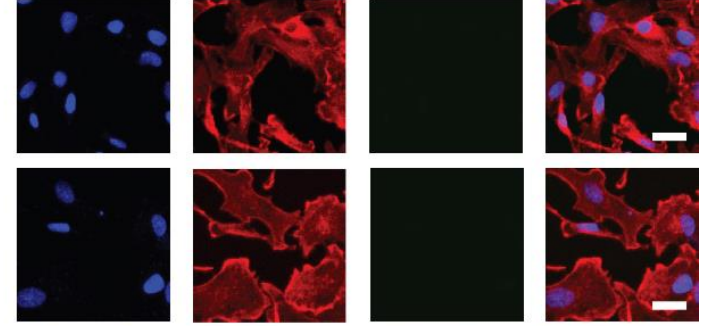

TAT-Lip
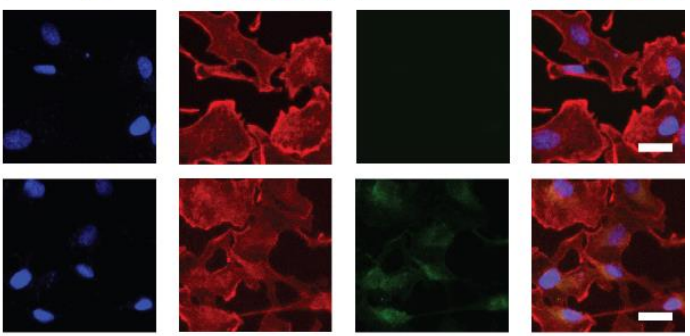

FA-TAT-Lip
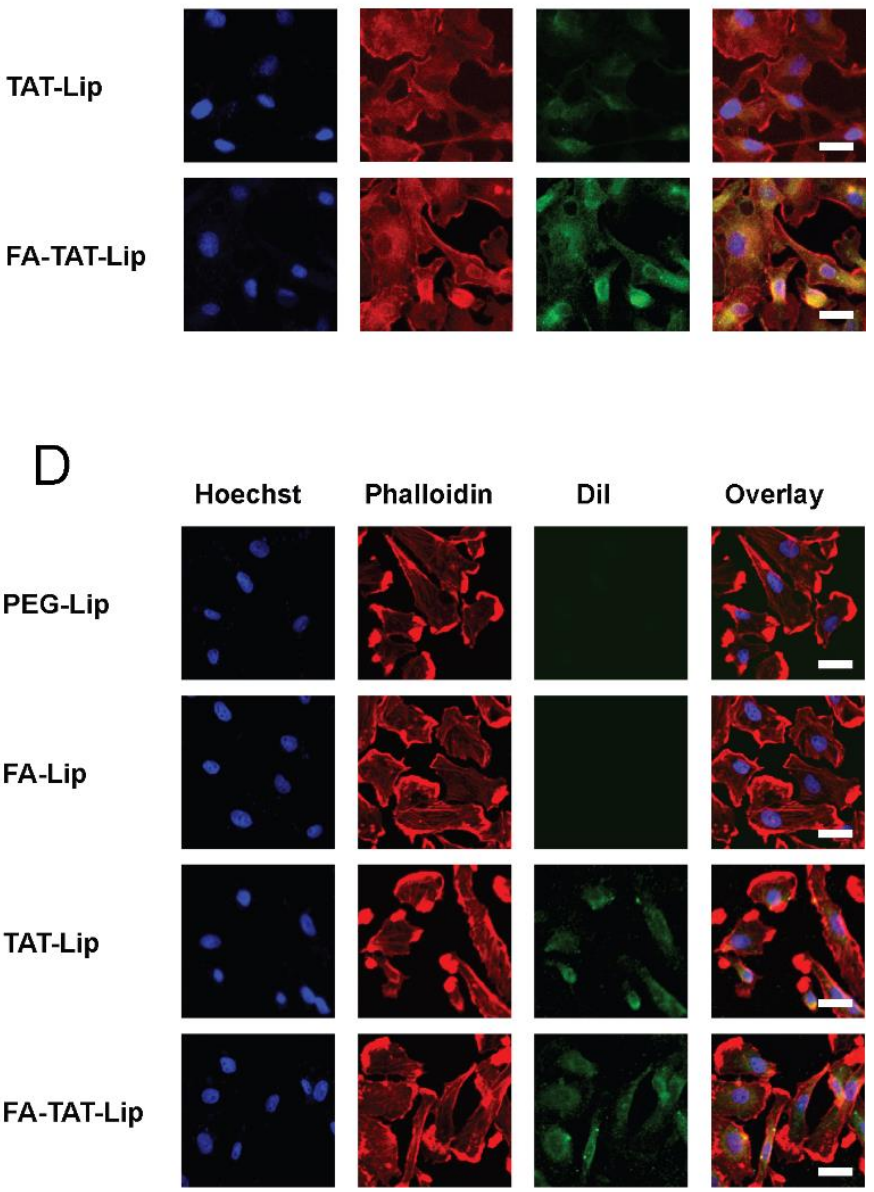

Overlay
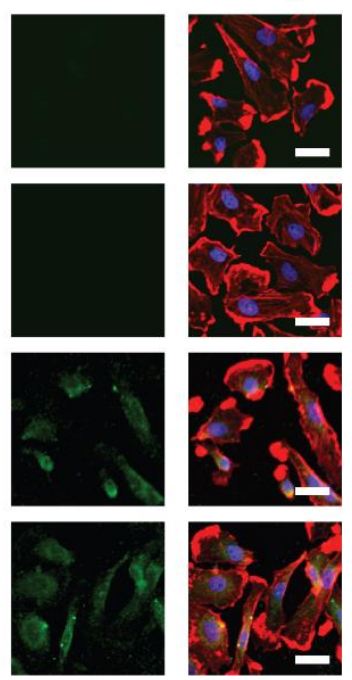

Fig. 3. (A) Optimized cellular uptake of FA-TAT-Lip on SKOV3 cells measured by flow cytometry. (B) Confocal microscopy observations of the uptake of Dil labeled liposomes by SKOV3 cells, showing a higher intracellular accumulation of FA-TAT-Lip. Hoechst, Phalloidin, Dil represent cell nuclei, cytoskeleton, and liposomes, respectively. (C) Cellular uptake of Dil labeled liposomes on MCF-7 cells measured by flow cytometry and (D) the corresponding confocal images of the cellular uptake on MCF-7 cells. Scale bars represent $20 \mu \mathrm{m} .{ }^{* * *}$ represents statistically significant difference $(p<$ 0.001 ), N.S. represents no statistically significant difference. (Mean $\pm S D, n=3$ )

3D tumor spheroid uptake and penetration

To recapitulate the $3 \mathrm{D}$ cell structure of solid tumors, we created a cost-effective 3D tumor spheroid model by cultivating cells in the non-attachment environment. Compared to 2D cell model, these tumor spheroids possess a 3D structure with the extracellular matrix, spatial 
geometry and hypoxic or necrotic regions [39] that can better mimic the real in vivo solid tumors. It is an alternative platform to evaluate the in vivo performance of the liposomes. The FR expression on the tumor spheroids was also detected, which confirms the difference of the FR expression on SKOV3 and MCF-7 tumor spheroids (Fig. S5B).

PEG-Lip, FA-Lip, TAT-Lip, and FA-TAT-Lip were incubated with SKOV3 or MCF-7 tumor spheroids for $4 \mathrm{~h}$ and then observed using CLSM. In consistent with the 2D cellular uptake, PEG-Lip has very limited SKOV3 and MCF-7 tumor spheroid uptake and internalization as indicated by the very low fluorescent signal (Fig. 4A). Adding the targeting ligand FA could not obviously increase the uptake while using the cell penetrating peptide TAT could dramatically increase the tumor spheroid accumulation. The combination of FA and TAT further promotes the tumor spheroid accumulation capability. Flow cytometry results show a $37 \%$ and $98 \%$ increase of the tumor spheroid uptake of the FA-TAT-Lip by the SKOV3 spheroid cells compared to the single ligand TAT-Lip and FA-Lip, respectively (Fig. 4B). However, this further increase only occurs on the FR positive SKOV3 spheroids but not the FR negative MCF-7 spheroids as shown in Fig. 4C and Fig. 4D. The results prove that the receptor-mediated synergistic effect also takes place in this $3 \mathrm{D}$ model.

A quantitative determination of the penetration capability of the liposomes was performed by analyzing the distribution of PEG-Lip, FA-Lip, TAT-Lip, and FA-TAT-Lip within the SKOV3 tumor spheroids at the depth of $80 \mu \mathrm{m}$. As shown in Fig. 4E, PEG-Lip and FA-Lip only display limited fluorescence intensity on the edge of the spheroid, indicating their low penetration capability. In contrast, TAT-Lip and FA-TAT-Lip penetrate much deeper as demonstrated by the much wider fluorescence distribution from edge to the center with a gradual decrease. Some of the fluorescence signal is even observed at the center area of the spheroids, indicating a high tumor spheroid penetration capability. Effective penetration of drugs or nanoparticles into solid tumors is critical for cancer treatment, but remains a challenge, especially for nanoparticles, due to the compact 3D structure of solid tumors as well as the elevated pressure at the center of tumors[40, 41]. As there is no much difference between the penetration pattern of FA-TATLip and TAT-Lip although FA-TAT-Lip has increased overall tumor spheroid uptake, we attribute the penetration capability mainly to the TAT peptide, which is in accordance with the previous study[42]. 
A

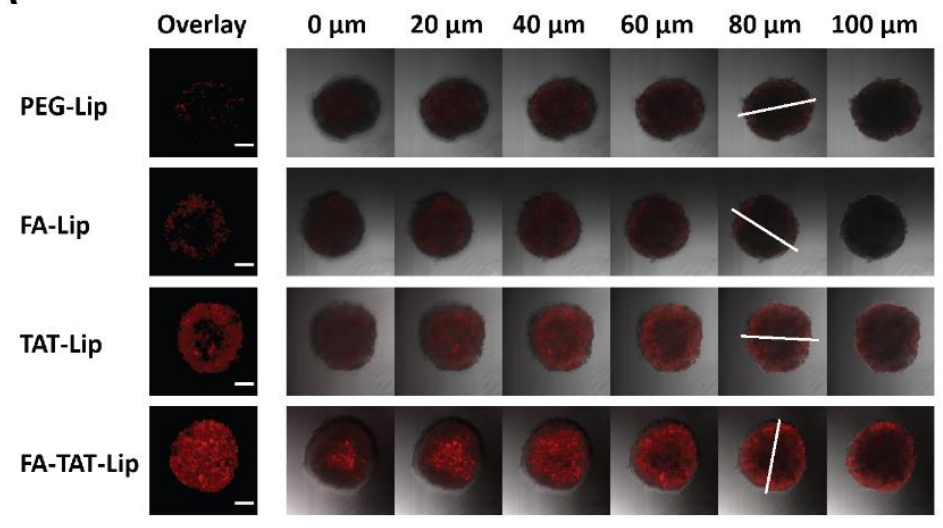

C

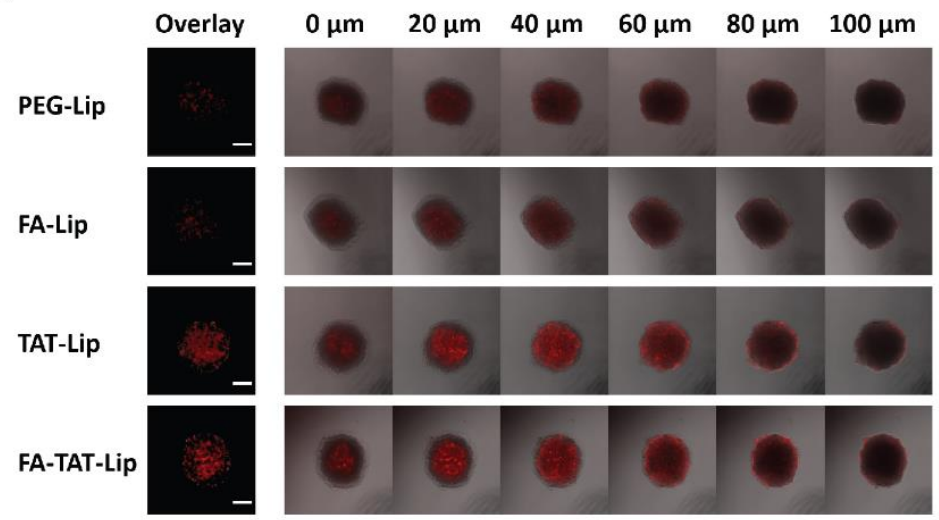

$\mathrm{B}$

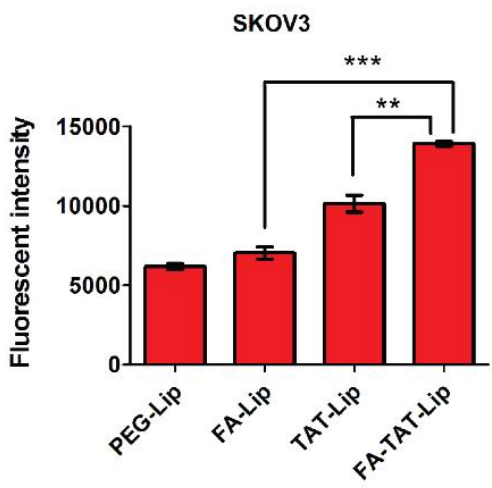

D

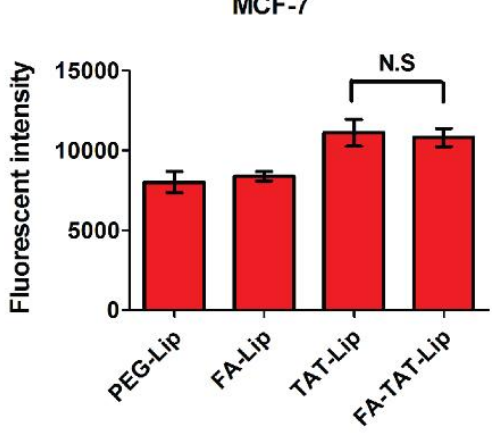

$E$

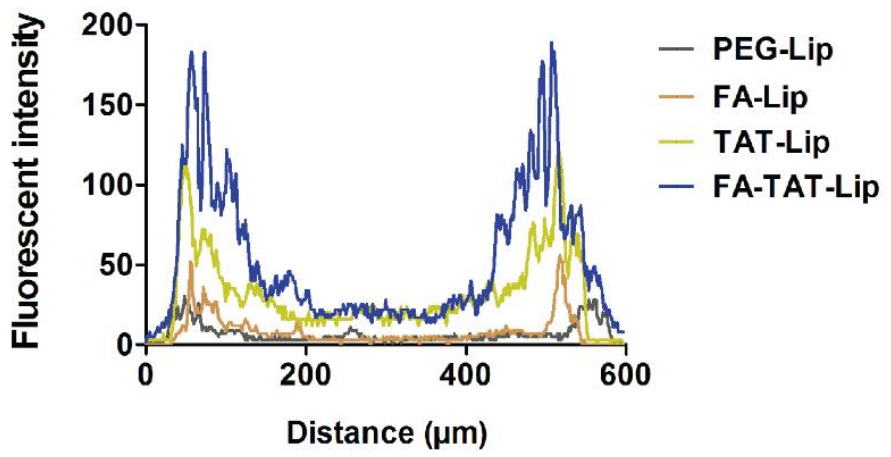

Fig. 4. (A) CLSM observation of overall and layer-by-layer images of Dil labeled liposomes in SKOV3 tumor spheroids. (B) Flow cytometry results of Dil labeled liposome uptake by SKOV3 tumor spheroids after dissociating the spheroids into single cells. The FA-TAT-Lip shows $37 \%$ and $98 \%$ increased uptake compared to the single ligand TAT-Lip and FA-Lip, respectively. (C) CLSM observation and (D) flow cytometry results of cellular uptake of Dil labeled liposomes in MCF-7 tumor spheroids. There is no significant difference between the FA-TAT-Lip and the TAT-Lip group. (E) Tumor spheroids penetration efficiency test by analyzing the Dil fluorescence intensity distribution along the line across the center of the SKOV3 tumor spheroids at the depth of $80 \mu \mathrm{m}$ (shown as a white line in Fig. 4A) of PEG-Lip, FA-Lip, TAT-Lip and FA-TAT-Lip. Scale bars represent 200 $\mu \mathrm{m} .{ }^{* *}, * * *$ represents statistically significant difference $p<0.01$ and $p<0.001$, respectively. N.S. represents no statistically significant difference (Mean $\pm S D, n=3$ ). 
In vivo tumor targeting and ex vivo tumor accumulation

To evaluate the in vivo targeting efficacy of the dual-ligand liposomes, the SKOV3 xenograft tumor model was established using nude mice. A near-infrared fluorescent dye DiR was utilized to track the liposomes as DiR could avoid the interference of the fluorescent signal from the body part of the mice. The time elapsed whole animal images of the liposomes biodistribution is shown in Fig. 5A. TAT-Lip and FA-TAT-Lip start to accumulate at the tumor site from $6 \mathrm{~h}$. At $24 \mathrm{~h}$, all of the four liposomes display the maximum tumor accumulation while the PEG-Lip and FA-Lip show weaker fluorescent signals than that of the TAT-Lip and FA-TAT-Lip. After $24 \mathrm{~h}$, the fluorescent signal starts to decrease. However, the fluorescent signal of FA-TAT-Lip decreases much slower than the other three liposomes and remains relatively strong even at $72 \mathrm{~h}$. Excised tumors from the mice are also imaged (Fig 5B). We could directly observe the prolonged tumor retention capability of FA-TAT-Lip. A quantitative comparison of the tumor site accumulation of the four formulations at different time points based on the fluorescence intensity is summarized in Fig 5C. Consistent with the images of the whole animal and the excised tumors, FA-TAT-Lip possesses sustained accumulation in the tumor site although it could not enhance the maximum tumor accumulation. At $72 \mathrm{~h}, \mathrm{FA}-\mathrm{TAT}$ Lip has $140 \%, 136 \%$ and $62 \%$ higher tumor accumulation than that of the PEG-Lip, FA-Lip, and TAT-Lip, respectively. The similar conclusion could also be drawn from the tumor to liver ratio analysis, shown in Fig. S7. Moreover, the biodistribution of the PEG-Lip, FA-Lip, TATLip and FA-TAT-Lip in the major organs (heart, liver, spleen, lung, kidney) and tumor at 6, 12, 24, 48 and $72 \mathrm{~h}$ were summarized in Fig. S8. We found that these four liposome formulations accumulated rapidly in the liver and spleen, and reached the maximum accumulation at 6 or $12 \mathrm{~h}$. In contrast, they accumulated gradually in the tumors and exhibited the maximum accumulation at $24 \mathrm{~h}$. Interestingly, although both the FA-TAT-Lip and TATLip (Fig. 5C) had similar accumulations in the tumor at $24 \mathrm{~h}$, the FA-TAT-Lip formulation's signal decreased much slower than the other three liposomes, implying a prolonged retention in the tumor site. The longer retention time of FA-TAT-Lip could be attributed to both FA and TAT. TAT increases the cell entry and the tumor penetration, leading to a higher and quicker accumulation than PEG-Lip and FA-Lip. FA provides sustained and strong interaction with the FR overexpressed tumor cells, therefore it takes a longer time to clear out theses liposomes from the tumors, compared to the TAT-Lip without FA. This in vivo result fully validates the 
advantage of utilizing the synergistic effect of the dual-ligand liposomes (FA-TAT-Lip) for enhanced targeting and prolonged retention in tumors.

The size and surface properties of the drug carrier have been well recognized as the most important factors affecting tumor accumulation in vivo. Circulating liposomes with a proper size could take advantage of the leaky vascular structure at the tumor site and extravasate into tumor tissue[43], which is referred as the EPR (enhanced permeability and retention) effect[44]. In our study, a proper size $(\sim 70 \mathrm{~nm})$, PEGylated surface, and slightly negative zeta-potential ensure a prolonged circulating time of the dual-ligand liposome (FA-TAT-Lip) in the blood, which is confirmed by the in vitro macrophage uptake test. Although the cationic peptide TAT may favor their clearance by the immune system[45], the major area of the liposomes is covered by PEG, which will shield some of TAT's positive charge and give FA-TAT-Lip a zetapotential comparable to PEG-Lip, thus ensuring a long blood circulation time. For the PEGLip and FA-Lip, although they could also accumulate at the tumor site to a certain extent, they could not be internalized into tumor cells efficiently and therefore be gradually drained away. This is in consistent with the finding that PEGylated liposomes preferentially accumulate in the perivascular areas and adjacent extracellular space[46]. 
A

PEG-Lip FA-Lip TAT-Lip FA-TAT-Lip

$2 \mathbf{h}$

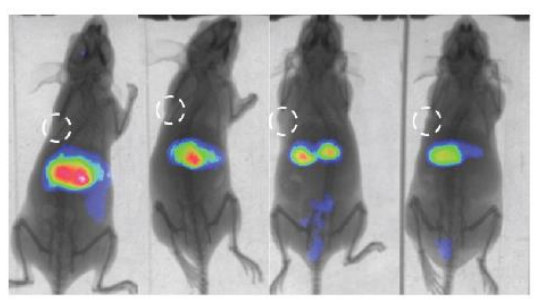

$12 \mathrm{~h}$

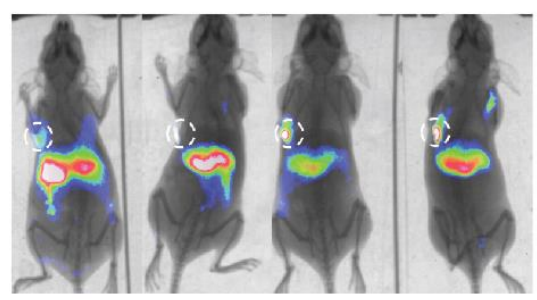

$48 \mathrm{~h}$

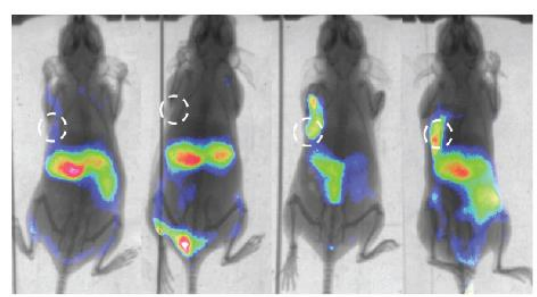

B

$6 \mathrm{~h}$

PEG-Lip FA-Lip TAT-Lip FA-TAT-Lip

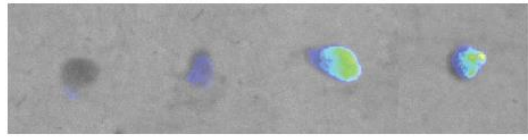

$24 \mathrm{~h}$

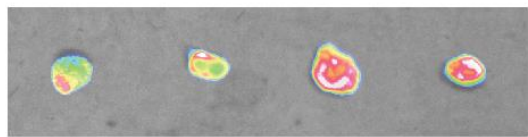

$72 \mathrm{~h}$

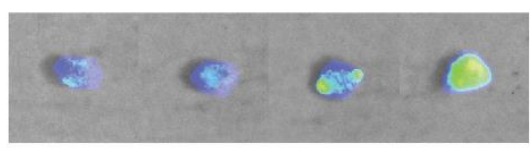

C

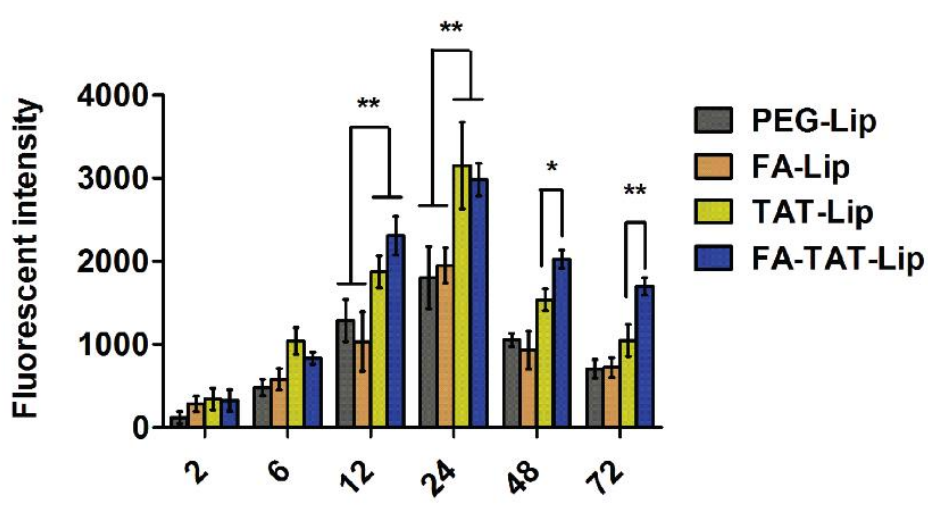

Time h

Fig. 5. (A) In vivo whole animal fluorescent images of the SKOV3 tumor-bearing mice at different time points after systemic administration of DiR-loaded liposomes. The dotted circles indicate the 
location of the tumors. Images show the fluorescent radiant intensity from the sample, and bars represent numeric radiant intensity analyzed by Image-J. FA-TA-Lip showed increased tumor accumulation before $24 \mathrm{~h}$ yet an obviously slowed decrease compared to other groups. (B) Fluorescent images of the excised SKOV3 tumors at different time points after injection of DiRloaded liposomes. Images show the fluorescent radiant intensity from the sample, and bars represent numeric radiant intensity analyzed by Image-J. FA-TAT-Lip still had a strong signal even after $72 \mathrm{~h}$. (C) Semi-quantified analysis of the mean fluorescent signal intensity of the tumor site in the whole animal images using Image-J, the background signal was exempted using the images at 0 h. ${ }^{*}, * *$ represents statistically significant difference $p<0.05$ and $p<0.01$, respectively. (Mean \pm SD, $\mathrm{n}=3)$.

\section{Conclusion}

A library of single-ligand and dual-ligand liposomes incorporating an active targeting ligand FA or/and a cell penetrating peptide TAT are synthesized using a combinatorial one-step microfluidic method. By adjusting the key parameter of the microfluidic method - flow rate ratio (FRR), the liposome size can be precisely controlled while the surface properties such as zeta-potential and ligand density remaining constant. The dual-ligand liposomes show increased monolayer cellular uptake and enhanced 3D tumor spheroid penetration due to a synergistic effect of FA and TAT. The animal study directly proved the increased tumor accumulation and prolonged retention of the dual-ligand liposomes. This one-step microfluidic method that synthesizes liposomes in a controlled manner in combination with using 2D, 3D and animal model has provided an attractive platform technology for accelerating the translation of functional liposomes into practical use and promoting the discovery of personalized treatment.

\section{Acknowledgement}

This project is supported by the Australian Research Council (ARC) Future Fellowship Project (FT140100726). Rui acknowledges Ph.D. scholarships from the University of Queensland. This work was performed in part at the Queensland node of the Australian National Fabrication Facility, a company established under the National Collaborative Research Infrastructure Strategy to provide nano and micro-fabrication facilities for Australia's researchers. The authors acknowledge the facilities, and the scientific and technical assistance, of the Australian Microscopy \& Microanalysis Research Facility at the Centre for Microscopy and Microanalysis, The University of Queensland. 


\section{References}

[1] T.M. Allen, P.R. Cullis, Liposomal drug delivery systems: from concept to clinical applications, Advanced drug delivery reviews, 65 (2013) 36-48.

[2] R.A. Petros, J.M. DeSimone, Strategies in the design of nanoparticles for therapeutic applications, Nat Rev Drug Discov, 9 (2010) 615-627.

[3] Y. Barenholz, Doxil (R) - The first FDA-approved nano-drug: Lessons learned, J Control Release, 160 (2012) 117-134.

[4] J.C. Kraft, J.P. Freeling, Z. Wang, R.J. Ho, Emerging research and clinical development trends of liposome and lipid nanoparticle drug delivery systems, Journal of pharmaceutical sciences, 103 (2014) 29-52.

[5] D. Bobo, K.J. Robinson, J. Islam, K.J. Thurecht, S.R. Corrie, Nanoparticle-Based Medicines: A Review of FDA-Approved Materials and Clinical Trials to Date, Pharmaceutical research, 33 (2016) 2373-2387. [6] V.P. Torchilin, Multifunctional, stimuli-sensitive nanoparticulate systems for drug delivery, Nat Rev Drug Discov, 13 (2014) 813-827.

[7] V.P. Torchilin, Multifunctional nanocarriers, Advanced drug delivery reviews, 64 (2012) 302-315.

[8] F. Perche, V.P. Torchilin, Recent trends in multifunctional liposomal nanocarriers for enhanced tumor targeting, Journal of drug delivery, 2013 (2013) 705265.

[9] X. Ying, H. Wen, W.L. Lu, J. Du, J. Guo, W. Tian, Y. Men, Y. Zhang, R.J. Li, T.Y. Yang, D.W. Shang, J.N. Lou, L.R. Zhang, Q. Zhang, Dual-targeting daunorubicin liposomes improve the therapeutic efficacy of brain glioma in animals, J Control Release, 141 (2010) 183-192.

[10] K. Takara, H. Hatakeyama, N. Ohga, K. Hida, H. Harashima, Design of a dual-ligand system using a specific ligand and cell penetrating peptide, resulting in a synergistic effect on selectivity and cellular uptake, Int J Pharmaceut, 396 (2010) 143-148.

[11] X.Q. Zhang, X. Xu, N. Bertrand, E. Pridgen, A. Swami, O.C. Farokhzad, Interactions of nanomaterials and biological systems: Implications to personalized nanomedicine, Advanced drug delivery reviews, 64 (2012) 1363-1384.

[12] A. Samad, Y. Sultana, M. Aqil, Liposomal drug delivery systems: an update review, Current drug delivery, 4 (2007) 297-305.

[13] P. Zhao, H. Wang, M. Yu, S. Cao, F. Zhang, J. Chang, R. Niu, Paclitaxel-loaded, folic-acid-targeted and TAT-peptide-conjugated polymeric liposomes: in vitro and in vivo evaluation, Pharmaceutical research, 27 (2010) 1914-1926.

[14] R. Niu, P. Zhao, H. Wang, M. Yu, S. Cao, F. Zhang, J. Chang, Preparation, characterization, and antitumor activity of paclitaxel-loaded folic acid modified and TAT peptide conjugated PEGylated polymeric liposomes, Journal of drug targeting, 19 (2011) 373-381.

[15] Y. Zhu, L. Cheng, L. Cheng, F. Huang, Q. Hu, L. Li, C. Tian, L. Wei, D. Chen, Folate and TAT peptide co-modified liposomes exhibit receptor-dependent highly efficient intracellular transport of payload in vitro and in vivo, Pharmaceutical research, 31 (2014) 3289-3303.

[16] C.X. Zhao, Multiphase flow microfluidics for the production of single or multiple emulsions for drug delivery, Advanced drug delivery reviews, 65 (2013) 1420-1446.

[17] C.X. Zhao, A.P.J. Middelberg, Microfluidic synthesis of monodisperse hierarchical silica particles with raspberry-like morphology, Rsc Adv, 3 (2013) 21227-21230.

[18] C.X. Zhao, L.Z. He, S.Z. Qiao, A.P.J. Middelberg, Nanoparticle synthesis in microreactors, Chemical Engineering Science, 66 (2011) 1463-1479.

[19] R. Ran, Q. Sun, T. Baby, D. Wibowo, A.P. Middelberg, C.-X. Zhao, Multiphase microfluidic synthesis of micro-and nanostructures for pharmaceutical applications, Chemical Engineering Science, (2017).

[20] P.M. Valencia, O.C. Farokhzad, R. Karnik, R. Langer, Microfluidic technologies for accelerating the clinical translation of nanoparticles, Nature nanotechnology, 7 (2012) 623-629. 
[21] A. Jahn, W.N. Vreeland, D.L. DeVoe, L.E. Locascio, M. Gaitan, Microfluidic directed formation of liposomes of controlled size, Langmuir : the ACS journal of surfaces and colloids, 23 (2007) 6289-6293. [22] R.R. Hood, D.L. DeVoe, High-Throughput Continuous Flow Production of Nanoscale Liposomes by Microfluidic Vertical Flow Focusing, Small, 11 (2015) 5790-5799.

[23] T. Baby, Y. Liu, A.P.J. Middelberg, C.X. Zhao, Fundamental studies on throughput capacities of hydrodynamic flow-focusing microfluidics for producing monodisperse polymer nanoparticles, Chemical Engineering Science, 169 (2017) 128-139.

[24] J.M. Harris, R.B. Chess, Effect of pegylation on pharmaceuticals, Nat Rev Drug Discov, 2 (2003) 214-221.

[25] J. Sudimack, R.J. Lee, Targeted drug delivery via the folate receptor, Advanced drug delivery reviews, 41 (2000) 147-162.

[26] V.P. Torchilin, Tat peptide-mediated intracellular delivery of pharmaceutical nanocarriers, Advanced drug delivery reviews, 60 (2008) 548-558.

[27] Q.Y. Zhang, J. Tang, L. Fu, R. Ran, Y.Y. Liu, M.Q. Yuan, Q. He, A pH-responsive alpha-helical cell penetrating peptide-mediated liposomal delivery system, Biomaterials, 34 (2013) 7980-7993.

[28] Y.Y. Liu, R. Ran, J.T. Chen, Q.F. Kuang, J. Tang, L. Mei, Q.Y. Zhang, H.L. Gao, Z.R. Zhang, Q. He, Paclitaxel loaded liposomes decorated with a multifunctional tandem peptide for glioma targeting, Biomaterials, 35 (2014) 4835-4847.

[29] A. Nagayasu, K. Uchiyama, H. Kiwada, The size of liposomes: a factor which affects their targeting efficiency to tumors and therapeutic activity of liposomal antitumor drugs, Advanced drug delivery reviews, 40 (1999) 75-87.

[30] J.M. Zook, W.N. Vreeland, Effects of temperature, acyl chain length, and flow-rate ratio on liposome formation and size in a microfluidic hydrodynamic focusing device, Soft Matter, 6 (2010) 1352-1360.

[31] J. Leng, S.U. Egelhaaf, M.E. Cates, Kinetics of the micelle-to-vesicle transition: Aqueous lecithinbile salt mixtures, Biophys J, 85 (2003) 1624-1646.

[32] C.B. He, Y.P. Hu, L.C. Yin, C. Tang, C.H. Yin, Effects of particle size and surface charge on cellular uptake and biodistribution of polymeric nanoparticles, Biomaterials, 31 (2010) 3657-3666.

[33] R. Ran, A.P.J. Middelberg, C.X. Zhao, Microfluidic synthesis of multifunctional liposomes for tumour targeting, Colloid Surface B, 148 (2016) 402-410.

[34] S.H. Boddu, R. Vaishya, J. Jwala, A. Vadlapudi, D. Pal, A. Mitra, Preparation and characterization of folate conjugated nanoparticles of doxorubicin using PLGA-PEG-FOL polymer, Med Chem, 2 (2012) 68-75.

[35] A. Gabizon, A.T. Horowitz, D. Goren, D. Tzemach, F. Mandelbaum-Shavit, M.M. Qazen, S. Zalipsky, Targeting folate receptor with folate linked to extremities of poly(ethylene glycol)-grafted liposomes: In vitro studies, Bioconjugate Chem, 10 (1999) 289-298.

[36] R. Molinaro, C. Corbo, J.O. Martinez, F. Taraballi, M. Evangelopoulos, S. Minardi, I.K. Yazdi, P. Zhao, E. De Rosa, M.B. Sherman, A. De Vita, N.E. Toledano Furman, X. Wang, A. Parodi, E. Tasciotti, Biomimetic proteolipid vesicles for targeting inflamed tissues, Nat Mater, 15 (2016) 1037-1046.

[37] R. Molinaro, M. Evangelopoulos, J.R. Hoffman, C. Corbo, F. Taraballi, J.O. Martinez, K.A. Hartman, D. Cosco, G. Costa, I. Romeo, M. Sherman, D. Paolino, S. Alcaro, E. Tasciotti, Design and Development of Biomimetic Nanovesicles Using a Microfluidic Approach, Adv Mater, 30 (2018).

[38] L.M. Pan, Q.J. He, J.N. Liu, Y. Chen, M. Ma, L.L. Zhang, J.L. Shi, Nuclear-Targeted Drug Delivery of TAT Peptide-Conjugated Monodisperse Mesoporous Silica Nanoparticles, J Am Chem Soc, 134 (2012) 5722-5725.

[39] R.M. Sutherland, Cell and Environment Interactions in Tumor Microregions - the Multicell Spheroid Model, Science, 240 (1988) 177-184.

[40] A.I. Minchinton, I.F. Tannock, Drug penetration in solid tumours, Nat Rev Cancer, 6 (2006) 583592. 
[41] I.F. Tannock, C.M. Lee, J.K. Tunggal, D.S.M. Cowan, M.J. Egorin, Limited penetration of anticancer drugs through tumor tissue: A potential cause of resistance of solid tumors to chemotherapy, Clin Cancer Res, 8 (2002) 878-884.

[42] L.Z. Zhang, Y.L. Zhou, G.C. Li, TAT peptide conjugated nanoparticles for enhanced tumor penetration, Nanomed-Nanotechnol, 12 (2016) 562-562.

[43] R.K. Jain, Transport of molecules across tumor vasculature, Cancer metastasis reviews, 6 (1987) 559-593.

[44] H. Maeda, Y. Matsumura, Tumoritropic and lymphotropic principles of macromolecular drugs, Critical reviews in therapeutic drug carrier systems, 6 (1989) 193-210.

[45] Y. Huang, Y. Jiang, H. Wang, J. Wang, M.C. Shin, Y. Byun, H. He, Y. Liang, V.C. Yang, Curb challenges of the "Trojan Horse" approach: smart strategies in achieving effective yet safe cell-penetrating peptide-based drug delivery, Advanced drug delivery reviews, 65 (2013) 1299-1315.

[46] D.B. Kirpotin, D.C. Drummond, Y. Shao, M.R. Shalaby, K. Hong, U.B. Nielsen, J.D. Marks, C.C. Benz, J.W. Park, Antibody targeting of long-circulating lipidic nanoparticles does not increase tumor localization but does increase internalization in animal models, Cancer Res, 66 (2006) 6732-6740. 